\title{
The Recent Star Formation History of the LMC Southwest Quadrant
}

\author{
Paolo Battinelli \\ Osservatorio Astronomico di Roma, Italia
}

Serge Demers

Université de Montréal, Canada

\section{Introduction}

Recently, Demers \& Battinelli (1998) have shown that the young intercloud stellar aggregates are 10 to $25 \mathrm{Myr}$ old, thus younger than the estimated age (200 Myr) of the last close LMC-SMC encounter (see, e.g., Irwin et al. 1996). These stellar aggregates show a distance gradient that confirms the existence of a "link" between the two Magellanic Clouds. Numerous blue stars have been detected by Demers \& Irwin (1991) east of the aggregate ICA76 (the closest to the LMC). These blue stars, that extend to the southwestern periphery of the LMC, may be an aftermath of the close LMC-SMC encounter. Our present study is aimed to survey this SW part of the LMC and to determine the origin of these blue stars.

\section{Observations}

Twenty one $15^{\prime} \times 15^{\prime}$ fields were observed, in B and V, with the CTIO $1.5 \mathrm{~m}$ telescope. The detector was a Tek 2K CCD. The data were obtained in December 1997, during three consecutive photometric nights. For each field two $450 \mathrm{~s}$ $\mathrm{B}$ and $\mathrm{V}$ exposures were taken. Data were analyzed with DAOPHOT II and ALLFRAME. This combination of detector (large field) and exposure times allows us to produce CMDs down to $\mathrm{V}=22 \pm 0.05$, i.e., well below the populous turnoff of the bulk of the old population of the LMC.

We selected our target fields mainly looking at the distribution of the blue stars on F033 and F032 ESO/SERC survey fields. The plate pairs have been scanned by the APM in order to produce a CMD of the area. A certain number of targets were also selected in order to survey the LMC radially (these fields are mostly along the $5^{h}$ hour circle).

\section{Results and Discussion}

Our survey indicates that there are essentially no young stars (age $<25 \mathrm{Myr}$ ) in the western periphery of the LMC. The stellar aggregate ICA $76-$ at $7^{\circ}$ from the center and with more than 80 blue stars - is to be considered unique. We have, however, found a region, about one degree in size, where numerous stars 
younger than $\sim 800 \mathrm{Myr}$ are seen. Some of them may be as young as $100 \mathrm{Myr}$. This region is not as close to the central part of the LMC as expected, but some 6 degrees from its center. These young stars are found in three of our CCD fields located near each other and close to ICA 76. The three fields with a substantial number of blue stars are just east of ICA 76 and between $-74^{\circ}$ and $-75^{\circ}$ (see Fig. 1). A detailed study of the stellar populations of this unusual area will be presented elsewhere:

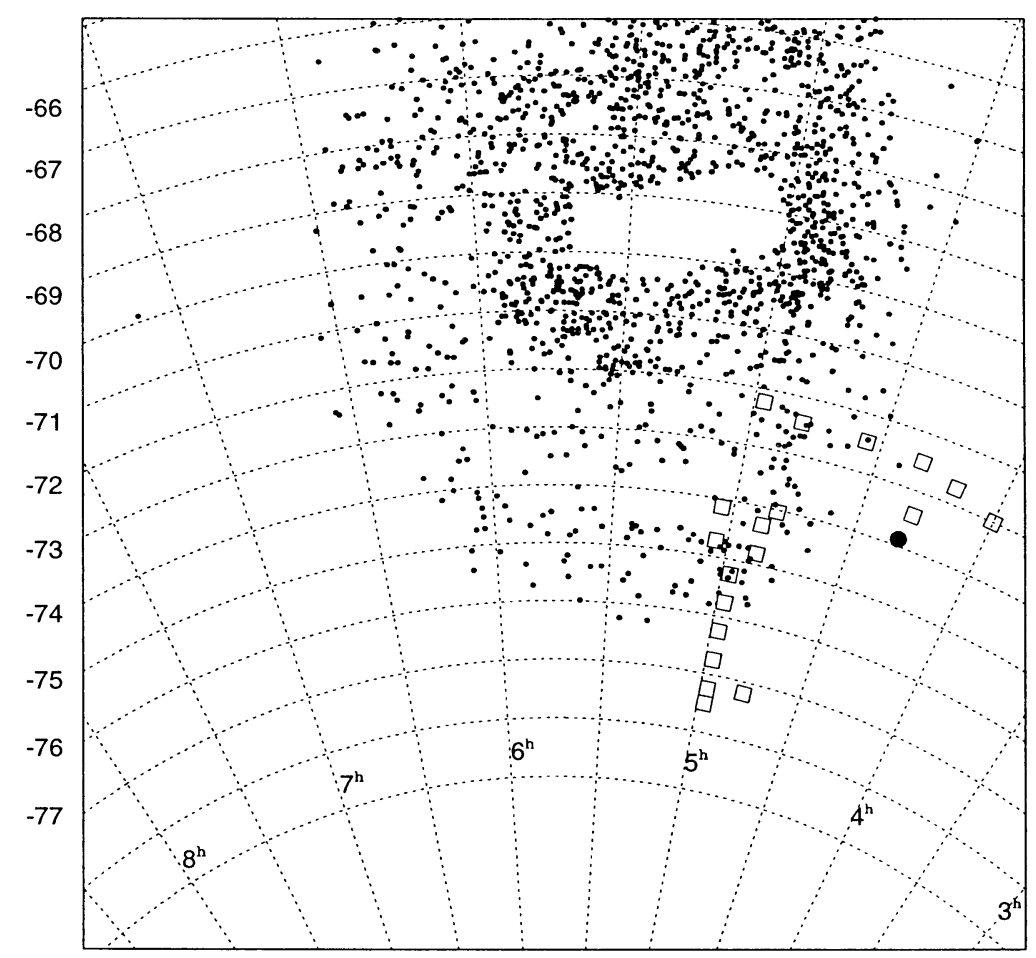

Figure 1. Dots: 1627 clusters from Kontizas et al. 1990; squares: CCD fields of the present survey; big black dot: ICA 76 . The three unusual fields are just East of ICA 76.

\section{References}

Demers, S., \& Battinelli, P. 1998, AJ, 115, 154

Demers, S., \& Irwin, M.J. 1991, A\&AS, 91, 171

Irwin, M.J., Demers, S., \& Kunkel, W.E. 1996, BAAS, 28, 932

Kontizas. M., Morgan, D.H., Hatzidimitriou, D., \& Kontizas, E. 1990, A\&AS, 84,527 